\title{
SAÚDE BUCAL E IMPLICAÇÕES ODONTOLÓGICAS DE PACIENTES PORTADORES DA DIABETES MELLITUS: REVISÃO DE LITERATURA
}

\author{
Oral Health and Dental Implications of Diabetic Patients: Literature Review
}

Gláucia de Almeida Caldeira ${ }^{\underline{1}}$, Maria Tereza de Oliveira e Souza $\underline{\underline{ }}$

\begin{abstract}
RESUMO
A Diabetes Mellitus é uma doença caracterizada pela não produção da insulina (tipo 1) ou pela incapacidade do corpo de absorvê-la de modo adequado. Pacientes diabéticos com níveis glicêmicos descontrolados são mais suscetíveis a doenças e infecções na cavidade oral. Este estudo trata-se de uma revisão de literatura cujo objetivo é abordar sobre as principais manifestações bucais em pacientes que sofrem de diabetes e a importância do acompanhamento odontológico. A pesquisa mostra que as manifestações da cavidade bucal mais frequentes nos portadores da DM são: xerostomia, hiposalivação, síndrome de ardência bucal, glossodinia, distúrbios da gustação, infecções, ulcerações na mucosa bucal, hipocalcificação do esmalte, perda precoce de dentes, dificuldade de cicatrização, doença periodontal, hálito cetônico e líquen plano, sendo a doença periodontal a mais frequente das queixas. Ainda, mostra a importância da realização de uma anamnese bem conduzida e os cuidados a serem tomados no pré e pós-operatório, uma vez que a diabetes causa dificuldades na cicatrização.

Palavras-chave: Diabetes mellitus. Manifestações bucais. Saúde bucal.
\end{abstract}

\section{ABSTRACT}

Diabetes Mellitus is a disease characterized by the non-production of insulin (type 1) or by the body's inability to absorb it properly. Diabetic patients with uncontrolled blood glucose levels are more susceptible to diseases and infections in the oral cavity. This study is a literature review aimed at addressing the main oral manifestations in patients suffering from diabetes and the importance of dental follow-up. Research shows that the most frequent manifestations of the oral cavity in DM patients are: dry mouth, hyposalivation, burning mouth syndrome, glossodynia, taste disorders, infections, ulcerations in the oral mucosa, enamel hypocalcification, early loss of teeth, difficulty in scarring, periodontal disease, ketone breath and lichen planus, with periodontal disease being the most frequent complaint. It also shows the importance of conducting a well-conducted anamnesis and the care to be taken pre- and postoperatively, since diabetes causes difficulties in healing.

Keywords: Diabetes mellitus. Oral manifestations. Oral health.

1. Acadêmica do Curso de Odontologia da Faculdade Morgana Potrich (FAMP), Mineiros - GO, Brasil.

2. Especialista em Periodontia. Docente da Faculdade Morgana Potrich (FAMP), Mineiros - GO, Brasil.

*Autor para Correspondência. E-mail: gcaldeiraodonto@gmail.com 


\section{INTRODUÇÃO}

A Diabetes Mellitus (DM) é uma doença metabólica crônica caracterizada pela hiperglicemia, isto é, a alta concentração de moléculas de glicose no sangue. Essa condição é resultado de um defeito na produção de insulina pelo pâncreas ou por sua má absorção. A insulina é um hormônio importante para o metabolismo que tem como uma de suas funções quebrar a glicose, transformando-a em energia. A DM representa um dos mais importantes problemas de saúde pública, tendo grande repercussão social e econômica, como também comprometendo a produtividade, qualidade de vida e sobrevida dos portadores de tal patologia. Pode ser classificada em tipo 1 (DM 1) e tipo 2 (DM 2). A DM1 é mais rara, quando comparada ao o número de portadores da DM2, e afeta com maior frequência crianças e adolescentes ${ }^{[1]}$.

A diabetes mellitus está diretamente ligada à saúde bucal, uma vez que é um fator sistêmico com manifestações na cavidade oral. Ela agrava ainda mais algumas doenças bucais já presentes em pessoas que não possuem uma saúde bucal satisfatória, como, por exemplo, a doença periodontal. Os distúrbios da cavidade bucal mais frequentes nos portadores da DM são: xerostomia, hiposalivação, síndrome de ardência bucal, glossodinia, distúrbios da gustação, infecções, ulcerações na mucosa bucal, hipocalcificação do esmalte, perda precoce de dentes, dificuldade de cicatrização, doença periodontal, hálito cetônico e líquen plano [2,3]. Quando se tem diabetes, necessita-se de maior atenção à saúde bucal e maior cautela dos profissionais de odontologia ao tratar esses pacientes. $\mathrm{O}$ diabetes mellitus abrange um grupo de distúrbios metabólicos que podem levar à hiperglicemia. Os principais sintomas são polidipsia, poliúria, polifagia e perda de peso ${ }^{[1]}$.

Com intuito de transmitir o conhecimento para qualquer tipo de público, mas em especial para acadêmicos e profissionais da área de odontologia, este trabalho objetiva, por meio de revisão de literatura, abordar sobre as principais manifestações bucais em pacientes que sofrem de diabetes e a importância do acompanhamento odontológico.

\section{METODOLOGIA}

A pesquisa foi desenvolvida através de metodologia de revisão de literatura, empregando o método explicativo, baseando-se na consulta a materiais didáticos, tais como livros, artigos científicos, monografias, periódicos, entre outros. A pesquisa foi realizada em três bases de dados sendo elas: PubMed, Google Acadêmico e Scielo, considerando os termos "saúde bucal", "implicações odontológicas", "paciente com diabetes", "distúrbios da mucosa oral em pacientes com DM". Foram utilizados sinônimos desses termos e a equivalência deles na língua inglesa. Ainda, foram excluídos os artigos publicados fora do período descrito e que não se restringissem ao tema e trabalhos que não tratavam da prevalência de distúrbios da mucosa oral em pacientes com DM. Assim, foram utilizados vinte e sete artigos publicados no período de 2012 a 2020 e excluídos artigos publicados fora do período descrito e que não se restringissem ao tema.

\section{REVISÃO DE LITERATURA}

Diabetes é o termo utilizado para descrever um grupo de doenças que tem como característica principal a não produção de insulina ou insuficiência desse hormônio, resultando em, por exemplo, elevação dos níveis de açúcar no sangue ${ }^{[4]}$. A DM é classificada em: tipo 1 que é o mais raro entre os dois tipos de diabetes e tem como característica principal a pouca produção de insulina pelo pâncreas, podendo afetar pessoas de todas as idades, porém sendo mais comum o desenvolvimento em crianças e adolescentes, e observada em apenas $10 \%$ na faixa etária adulta. Esse tipo de diabetes tem seus sintomas expostos de forma rápida; e o tipo 2 que é mais comum, representando 90 a $95 \%$ dos casos da doença. Existem sintomas comuns para os tipos 1 e 2 que incluem poliúria, sede, visão turva e cansaço, porém a DM2 se difere na evolução de seus sintomas, que são desenvolvidos mais lentamente, sendo mais frequentemente observada em pacientes adultos e idosos, apesar de crescente número de casos também em pessoas mais jovens. É válido salientar que o DM tipo 2 afeta, em sua maioria, indivíduos que apresentam excesso de peso. Os sintomas clássicos apresentados por portadores do Diabetes Mellitus são: poliúria, polidipsia, polifagia, emagrecimento e, nos casos mais graves, desidratação, acidose e distúrbios no nível de consciência. Assim, quando o indivíduo apresenta os sintomas, o diagnóstico é facilitado, porém, nem sempre os sintomas são visíveis, sendo necessária, então, uma investigação laboratorial minuciosa ${ }^{[5,6]}$.

A hiperglicemia trata-se de um termo que é utilizado pelos médicos para descrever a alta taxa glicose no sangue, acima de $126 \mathrm{mg} / \mathrm{dl}$ em jejum e acima de $200 \mathrm{mg} / \mathrm{dl}$ até duas horas após uma refeição, de pessoas que sofrem diabetes do (tipo 1 e 2) e pode ser causada por diversos fatos não necessariamente ligados à diabetes, cujos sintomas podem ser: fome excessiva, visão borrada, sede, perda de peso e aumento de frequência urinária. $\mathrm{O}$ tratamento torna-se necessário, pois pode evoluir para outros quadros, tendo como exemplo a cetoacidose diabética que causa respiração 
ofegante, aumento da frequência cardíaca, náusea, vômito, dentre outros. Já hipoglicemia acontece quando existe a baixa concentração de glicose presente no sangue, falando mais especificamente, quando a quantidade de glicose no sangue fica abaixo de $70 \mathrm{mg} / \mathrm{dL}$. Pode se diferenciar em dois tipos, a jejum e a pós-prandial (ou reativa), que ocorre depois das refeições e atua tanto em portadores quanto não portadores de diabetes ${ }^{[7]}$.

Dentre as manifestações bucais que podem acometer o paciente com DM não controlado, estão: Hipossalivação; Xerostomia; Glossodinia (infecção ou inflamação da língua); Hipocalcificação do esmalte; Halitose; Infecções, Distúrbios da gustação; Líquen plano; Ulcerações na mucosa bucal; Doença periodontal; e Dificuldade de cicatrização ${ }^{[4,8]}$. Há uma discussão dos estudiosos acerca da cárie. Alguns autores não encontram relação entre a doença cárie e a DM ${ }^{[5,7]}$, assim como outros autores dizem também que os pacientes portadores da DM, que não monitoram seu estado, não tratam a doença, apresentam resultados desfavoráveis nos índices CPO-D (Dente Cariado/ Perdido/ Obturado) ${ }^{[4,8]}$. Isso se dá por não existirem muitas provas de que uma dieta com pouca sacarose resulte na redução da ocorrência de cárie, pois a flora bacteriana do paciente portador de DM pode estar alterada, predispondo à doença cárie ${ }^{[4]}$.

A hipossalivação trata-se da diminuição ou a cessação do fluxo salivar. A principal defesa bucal é a saliva; ela possui diversos componentes como enzimas digestivas, eletrolíticos, lubrificantes, sistema tampão, glicoproteínas e proteínas antimicrobianas. Vale lembrar que mudanças em sua quantidade e qualidade afetam severamente a saúde oral [9]. Em pacientes com DM, as alterações na saliva provocam a redução da atividade antibacteriana, fúngica e viral; a redução da lubrificação, da lavagem e limpeza dos tecidos orais; além de dificuldades na digestão e fala ${ }^{[9]}$.

O controle da produção e do fluxo salivar é realizado pelo sistema nervoso autônomo, por meio de sua ação no neurotransmissor colinérgico acetilcolina, sendo um problema bucal frequentemente relatado pelos pacientes com DM [10]. A Xerostomia muitas vezes é utilizada como sinônimo da hipossalivação e caracterizada como a sensação de boca seca. A sensação de boca seca não está essencialmente relacionada à redução da quantidade de saliva. A xerostomia, além do desconforto, está relacionada com a candidíase oral, fissuras na mucosa oral, halitose, o aumento de cáries dentárias, sialoadenite (infecção das glândulas salivares), inflamação e/ou ulceração da língua e da mucosa bucal e queilite (inflamação e fissuras dos lábios). Uma grande parte dos pacientes com DM relatam possuir xerostomia ${ }^{[11]}$. Quando os pacientes com DM estão com um descontrole metabólico, a xerostomia e a hipossalivação costumam apresentar um agravamento, pois a desidratação ocasiona um aumento dos gradientes osmóticos dos vasos sanguíneos, o que compromete as glândulas salivares e, como resultado, a secreção da saliva é limitada ${ }^{[12]}$.

A candidíase oral é uma infecção causada pelo fungo Candida albicans, que frequentemente acomete pacientes com DM, dentre as causas está a redução do fluxo salivar e o estado imunocomprometido dos pacientes. É importante ressaltar que a simples presença do fungo Candida albicans não necessariamente seja um problema, a não ser que haja alterações químicas na cavidade oral. Isso porque a infecção pode aparecer como reação do uso inadequado de medicamentos químico terapêuticos, anti-histamínicos ou antibióticos. Além da diabetes, a idade, o uso de drogas, deficiências imunitárias, a má nutrição, hiperglicemia e a disfunção salivar tendem a contribuir para o surgimento da candidíase ${ }^{[9,11,13]}$.

A glossodinia (síndrome de ardência ou queimação na língua), causa uma sensação de ardor que pode ser ou não acompanhada de dor na língua ou em outra área da cavidade oral. Trata-se de uma doença que possui um tratamento complexo, utilizando-se de medicações que estimulem a salivação. Há diversos fatores que estão relacionados à origem da glossodinia e, entre as causas locais, cita-se: traumas, fungos, bactérias, desordens salivares, lesões na mucosa e outros. Entre os fatores sistémicos estão: desordem na glândula da tireoide, menopausa, doenças autoimunes, Diabetes Mellitus e outros. Pacientes com DM costumam apresentar a glossodinia, uma vez que essa condição causa um grande desiquilíbrio na produção de saliva ${ }^{[14]}$.

Halitose é a expressão comumente empregada para se referir ao odor desagradável oriundo da cavidade oral, que tem como causas fatores extra ou intraoral. Essa condição pode trazer sérios prejuízos na qualidade de vida do paciente pelo desconforto causado, tendo como causas doenças sistémicas, consumo abusivo de álcool e drogas, ou condições psicológicas que levem a uma redução do fluxo salivar ${ }^{[15,16]}$. A principal etiologia da halitose é alteração na microbiota oral, onde bactérias colonizam a língua por meio de um biofilme, os quais dão origem a compostos sulfúreos voláteis, ácidos orgânicos e aminas, a presença desse odor desagradável está comumente presente em pacientes com DM. A saliva exerce um papel fundamental no equilíbrio da microbiota oral e os diabéticos relatam constantemente sofrerem os efeitos desse desequilíbrio em sua cavidade oral [16]

Outra queixa frequente dos pacientes com DM são os distúrbios na degustação, que têm como fatores as doenças 
endócrinas e metabólicas, e a disfunção salivar é uma das causas que mais contribuem para o surgimento de alterações no paladar. Os pacientes relatam sensação de gosto alterado ou sabor azedo. Os portadores de DM com déficit no controle glicémico, ou que sofrem de neuropatia, possuem uma incidência maior a sofrer de distúrbios do paladar ${ }^{[8,17]}$.

A hipocalcificação do esmalte surge em decorrência de alterações durante a fase de mineralização ou da maturação da matriz orgânica. A hipocalcificação pode se manifestar como variações na translucidez ou mesmo a opacidade do esmalte (nessa há alterações na coloração do esmalte, mas não há perda do mesmo). Existem diversas condições que favorecem o surgimento da hipocalcificação, como por exemplo condições clínicas relacionadas a fatores químicos ou patológicos, nutricionais, neurológicos, bem como metabólicos como a Diabetes Mellitus. Manifestações orais, como distúrbios na degustação e a hipocalcificação, são normalmente mais diagnosticadas em pacientes diabéticos em comparação a pacientes não diabéticos ${ }^{[18]}$.

A Doença Periodontal (DP) trata-se de uma patologia que possui uma evolução contínua, tendo períodos de remissão e de agravamento, proveniente de uma ação inflamatória e imune do hospedeiro, mediante existência de bactérias anaeróbicas gram-negativas. É tida como um dos acometimentos mais frequentes em pacientes com DM, além de ser a segunda maior patologia dentária a afetar os humanos [19]. A nomenclatura dessa patologia é um termo genérico, visto que a DP compreende uma série de alterações que se desenvolvem no periodonto (engloba a gengiva, osso alveolar, cemento e o ligamento periodontal). Essa doença possui um desenvolvimento progressivo, tendo fases iniciais e avançadas ${ }^{[20]}$. Gengivite é descrita como a fase inicial da DP, sendo definida como um estado inflamatório em decorrência da existência de bactérias na margem gengival, podendo ocorrer sangramento e recessão da gengiva. A gengivite pode se agravar ainda mais, evoluindo para a periodontite ${ }^{[21]}$, tem como características o rubor, edema e a ausência de perda de aderência o que provoca um aumento na sensibilidade, podendo se apresentar na forma induzida por placa e não induzida por placa ${ }^{[22,23]}$.

Quando não tratada, a gengivite pode evoluir para periodontite e tal patologia acomete os tecidos periodontais, ocasiona uma destruição de fibras do ligamento periodontal e óssea, resultando na formação de bolsas com líquido purulento. Esse agravamento é causado pela presença de bactérias na placa, podendo levar a perdas dentárias ${ }^{[24]}$. A doença periodontal mostra uma etiologia com diversos fatores que contribuem para o seu desenvolvimento, como por exemplo a diabetes mellitus, o tabagismo, má higienização oral, alterações hormonais durante a gravidez e menopausa, alterações metabólicas nutricionais, imunossupressão entre outros [25]. Diferentes fatores associados ao DM podem influenciar a progressão e agressividade da DP: tipo de diabetes (mais comum no tipo 1), idade do paciente (aumento do risco durante e após a puberdade), maior duração da doença e controle metabólico inadequado [25, 26]. O diabetes está associado à maior severidade da doença periodontal, é decorrente do crescente acúmulo de produtos finais de glicosilação (AGEs) no plasma e nos tecidos, o diabetes é um dos fatores sistêmicos, que agravam a resposta do periodonto à placa bacteriana por meio de diversas modificações no organismo de ordem: bioquímica, genética e ambiental, imunológica ou tecidual [22,23].

O líquen plano é mais comum em pacientes com diabetes do tipo 1; estudos sugerem que isso ocorra pelo fato da diabetes do tipo 1 ser uma doença autoimune, assim como o líquen plano que possui um mecanismo de ação autoimune [11,12]. O diagnóstico do líquen plano é obtido por meio da análise geral das características da lesão em conjunto com exame histopatológico realizado por biópsia. O tratamento mais convencional é o uso de paliativos, visto que a cura não ocorre em todos os casos. Essa patologia pode se apresentar de diversas formas como: líquen plano reticular, erosivo, placa, papular, atrófico e bolhoso. Dentre essas manifestações as mais frequentes são o líquen plano reticular que possui estrias com aparência rendilhada, e o erosivo. Alguns estudos indicam que o aparecimento das lesões nos pacientes possa estar ligado a fatores psicológicos, como por exemplo o estresse ${ }^{[11,12]}$.

A cicatrização de tecidos trata-se de um complexo processo biológico que abrange a inflamação, proliferação e remodelação de toda a área afetada. Esse processo pode ser comprometido por causas sistêmicas ou locais que podem prejudicar uma ou mais etapas do procedimento da cicatrização. Pacientes com DM possuem uma cicatrização extremamente lenta, se comparada a pacientes não portadores de diabetes, pois existe uma incapacidade do organismo em responder às lesões sofridas, demorando a desenvolver uma resposta inflamatória, o que gera um desiquilíbrio na cicatrização geral. As alterações nos níveis glicêmicos causam complicações vasculares, levando a uma deficiência na macro e microcirculação local, resultando em danos nos tecidos e células. Dessa forma, a cicatrização é retardada através do decréscimo da nutrição e oxigenação dos tecidos lesionados ${ }^{[26]}$.

A cavidade oral apresenta uma grande quantidade e diversidade de microrganismos, o que favorece a entrada de 
bactérias na corrente sanguínea durante o tratamento odontológico. Esse quadro, no paciente portador de diabetes mellitus, requer muita atenção do cirurgião dentista, tendo em vista as complicações que o paciente pode apresentar. A profilaxia antibiótica tem como objetivo a redução do risco de infecção em sítio cirúrgico, não tendo papel preventivo pós-cirúrgico. Assim, deve ser iniciada antes do procedimento cirúrgico, visando a que o medicamento tenha concentração máxima no sangue durante o processo e continue atuando até que ocorra a cicatrização adequada dos tecidos. É necessário que o cirurgião-dentista conheça os medicamentos utilizados por pacientes com DM, uma vez que uma grande parte destes apresentam reações adversas, se utilizados com outros medicamentes normalmente adotados na prática odontológica. Há dificuldades na cicatrização devido à insuficiência vascular periférica, além de mudanças fisiológicas que contribuem para a redução da imunidade do paciente, aumentando os casos de infecções ${ }^{[8]}$.

Normalmente as próprias defesas do organismo conseguem controlar esse processo, porém os pacientes com DM podem apresentar complicações sistêmicas, sendo assim necessário utilizar uma droga bactericida dirigida contra microrganismos comumente encontrados na cavidade bucal [8]. É importante salientar que a profilaxia antibiótica só se torna necessária quando o paciente está com a diabetes descompensada. Caso seja necessário que um paciente com diabetes seja submetido a uma cirurgia odontológica, é importante que a alimentação seja equilibrada próxima à data da mesma, como também tomar as medicações de forma correta, tornando assim o procedimento bem mais seguro. Mesmo com esses e outros cuidados, o cirurgião dentista deve ficar atento aos sinais sistêmicos do paciente, tendo em vista que as complicações agudas do diabetes mellitus são bastante significativas e devem sempre ser consideradas durante o atendimento odontológico, como por exemplo a cetoacidose diabética, o estado hiperglicêmico hiperosmolar e a hipoglicemia. Em casos de hiperglicêmia hiperosmolar e os de cetoacidose apresentam elevada letalidade ${ }^{[27,28]}$. Da mesma forma que os cuidados são necessários antes da cirurgia, o pós-cirurgia também requer cuidados, incluindo alimentação fria, compressas geladas no local da cirurgia, além de manter uma boa higienização do local. Se mesmo com todos os cuidados, o paciente sentir incômodo, deve procurar o dentista para maiores esclarecimentos ${ }^{[28]}$.

O emprego de uma anamnese bem conduzida pelo cirurgião-dentista é uma conduta odontológica essencial a ser realizada no atendimento de pacientes com DM. Assim, uma avaliação deve ser feita durante a consulta inicial desse paciente, levantando dados sobre os hábitos alimentares do paciente, histórico de peso, apetite recente, idade, fumo, histórico familiar e o nível de urina o qual é primordial para um diagnóstico de paciente com DM ${ }^{[4,29]}$. O paciente que já faz tratamento relacionado ao diabetes mellitus deve ser questionado sobre quanto tempo tem a doença, ocorrência de hiperglicemias, histórico de diabetes que seguem um regime terapêutico e fazem tratamento regularmente podem ser tratados de maneira similar ao paciente não portador da DM, o que facilita os procedimentos dentários de rotina. Segundo, visando reduzir a tensão, os pacientes portadores de diabetes devem ser atendidos em consultas curtas pela parte da manhã com técnicas de sedação auxiliar quando apropriadas ${ }^{[8]}$. O paciente com DM pode ser assintomático, assim, durante um atendimento odontológico, é importante que o profissional esteja atento ao momento da anamnese. Em diversas ocasiões, é por meio do reconhecimento de fatores de risco para a doença, que se obtém uma suspeita clínica para a doença ${ }^{[8]}$.

\section{CONSIDERAÇÕES FINAIS}

O presente estudo mostrou que, além da doença periodontal, que é uma das queixas mais comuns dos diabéticos, existe uma vasta quantidade de malezas que podem acometê-los como xerostomia, hiposalivação, síndrome de ardência bucal, glossodinia, distúrbios da gustação, infecções, ulcerações na mucosa bucal, hipocalcificação do esmalte, perda precoce de dentes, dificuldade de cicatrização, hálito cetônico, candidíase oral e líquen plano. A grande maioria dos diabéticos apresenta algumas dessas manifestações quando comparados com pacientes que não tem DM.

$\mathrm{O}$ estudo mostrou ainda que o profissional da odontologia deve estar atento às queixas de seus pacientes, para isso, devem realizar uma boa anamnese, tanto para saber se seu paciente com DM possui alguma manifestação bucal, ou se está tomando algum medicamento que possa interferir com algum fármaco utilizado durante os procedimentos odontológicos, ou mesmo para descartar ou levantar alguma suspeita de DM em pacientes que não possuem diagnóstico confirmado da doença. Além disso, os profissionais da saúde bucal devem informar seus pacientes com DM ou com suspeita da doença, da importância da manutenção da saúde bucal, muitos pacientes diabéticos desconhecem a relação da doença com a saúde bucal.

\section{REFERÊNCIAS}

1. OLIVEIRA, T. F. de; MAFRA, R. P.; VASCONCELOS, M. G.; VASCONCELOS, R. G. Conduta Odontológica em Pacientes Diabéticos 
Considerações Clínicas. Artigo de revisão - Odontol. Clín.-Cient., Recife, [periódico online]. 2016. 15(1) $13-17$, jan./mar., B.

2. POUDEL, P.; GRIFFITHS, R.; WONG, V. W.; ARORA, A.; GEORGE, A. Knowledge and practices of diabetes care providers in oral health care and thei potential role in oral health promotion: A scoping review. Diabetes Res Clin Pract. [periódico online]. 2017 Aug; 130:266-277. doi:

10.1016/j.diabres.2017.06.004. Epub 2017 jun 12.

3. NAZIR, A. M.; ALGHAMDI, L.; ALKADI, M.; ALBEAJAN, N.; ALRASHOUDI, L.; ALHUSSAN, M. The burden of Diabetes, Its Oral Complications and Their Prevention and Management. [periódico online]. 2018 aug 15;6(8):1545-1553. doi: 10.3889/oamjms.2018.294. eCollection 2018 aug 20.

4. OLIVEIRA, M. de F.; DAMO, N. G.; RAITZ, I. W.; VEIGA, M. L. da; PEREIRA, L. Cuidados Odontológicos em Pacientes diabéticos. Artigo de revisão - Arq. Catarin Med., Blumenau. [periódico online]. 2019. 48(3):158170, jul./set.

5. SOUZA, M.; VIEIRA, A. J.; RAMOS, B. S.; COUTO, D. P. Conhecimento e conduta de médicos frente à interrelação diabetes mellitus e doença periodontal - Artigo de revisão - Centro Universitário de Anápolis -

UniEVANGÉLICA, [periódico online]. 2019.

6. LABOLITA, K. A.; SANTOS, I. B.; BALBINO, V. C.; ANDRADE, G. L. ARAUJO, I. C.; FERNANDES, D. C. Assistência Odontológica à Pacientes Diabéticos. Ciências Biológicas e de Saúde Unit, Alagoas, [periódico online] 2020. v. 6, n. 1, p. 89-98, abril.

7. ESTEVES, C.; NEVES, C.; CARVALHO, D. A Hipoglicemia no Diabético: Controvérsia na Avaliação, à Procura de suas Implicações. Rev. Acta Médica Portuguesa 25 (6), [periódico online]. 2012.

8. SILVA, E. T. C. da; VASCONCELOS, R. G.; MARINHO, S. A.; VASCONCELOS, M. G. Diabetes na odontologia: manifestações bucais e Condutas para atendimento. SALUSVITA, Bauru, [periódico online] 2020.v.39, n. 3, p. 877-901.

9. SILVA, D. F. B.; SILVA, J. E. da; SOUZA E. V. B. de; ALBUQUERQUE, C. R. J. de; CATÃO, M. H. C. de V. Alterações bucais decorrentes do Diabetes Mellitus Tipo 2. FOL -Faculdade de Odontologia de Lins/Unimep. 27(2) 2735. jul.-dez. 2017 ISSN Impresso: 0104-7582. ISSN Eletrônico: 2238-1236.

10. NICOLAU, J.; SIMÕES, A.; NOGUEIRA, F. N.; Diabetes: noções gerais para o Cirurgião-Dentista. Rev. Assoc. Paul. Cir. Dent; jul.-set. [periódico online] 2015; 69(3): 260-265.

11. TRETIN, M. S.; VERARDI, G.; FERREIRA, M. de C.; CARLI, J. P. de; SILVA, S. O. da; LIMA, I. F. P.; PARANHOS, L. R. Most Frequent Oral Lesions in Patients with

Type 2 Diabetes Mellitus. The Journal of Contemporary Dental Practice, Febuary [periódico online]. 2017;18(2):107-111.

12. YAMASHITA, J. M.; MOURA-GREC, P. G. de; CAPELARI, M. M.; SALES-PERES, A.; SALES-PERES, S. H. de C. Manifestações bucais em pacientes portadores de Diabetes

Mellitus: uma revisão sistemática. Rev Odontol UNESP. [periódico online]. 2013 May-June; 42(3): 211-220.

13. GOMES, C. C.; GUIMARÃES, L. S.; PINTO, L. C. C.; CAMARGO, G. A. da C. G.; VALENTE, M. I. B.; SARQUIS, M. I. de M. Investigations of the prevalence and virulence of Candida albicans in periodontal and endodontic lesions in diabetic and normoglycemic patients. J. Appl. Oral Sci. 25 (3). MayJun 2017

14. GONZÁLES-SERRANO, J.; SERRANO, J.; LÓPEZ-PINTOR, R. M.; PAREDES, V. M.; CASAÑAS, E.; HERNÁNDEZ, G. Prevalência de distúrbios da mucosa oral em pacientes com diabetes mellitus em comparação com um grupo controle. [periódico online]. 2016 | Artigo ID 5048967

15. MOKEEM, S. A. Halitosis: A Review of the Etiologic Factors and Association with Systemic Conditions and its Management. The Journal of Contemporary Dental Practice, [periódico online]. 2014. 15(6), 806-811.
16. FREITAS, A. I. J. de. A Abordagem do Médico Dentista ao Paciente Diabético. 74 f. Dissertação (Mestrado). 2015. Instituto Superior de Ciências da Saúde Egas Moniz.

17. ARANA, C.; MORENO-FERNÁNDEZ, A. M.; GÓMEZ-MORENO, G.; MORALES-PORTILHO, C.; SERRANO-OLMEDO, I.; MAYOR, M. C. de la C.; HERNÁNDES, T. M. Incremento de los parámetros de estrés oxidativo salival en pacientes con diabetes tipo 2: relación con la enfermedad periodontal. Endocrinología, Diabetes y Nutrición. [periódico online]. 2017. v. 64, Issue 5, May, p.258-264.

18. ANDRADE, C. E. de S.; LIMA, I. H. L.; SILVA, I. V. dos S. VASCONCELOS, M. G.; VASCONCELOS, R. G. As Principais Alterações Dentárias de Desenvolvimento. SALUSVITA. [periódico online]. 2017. Bauru, v. 36, n. 2 , p. $533-563$

19. SARMENTO, M. das G. S. Avaliação da Saúde Bucal de Pacientes Portadores de Diabetes Mellitus Tipo II Atendidos na UBS DR. José Figlioulo -Distrito Norte- Manaus/AM.2019. 110 f. Dissertação (Mestrado). Fundação Oswaldo Cruz, Manaus - AM. 2019.

20. KINANE, D. F.; STATHOUPOULOU, P. G.; PAPAPANOU, P. N Periodontal Diaseases. Nat Rev Dis Primers. 2017 Jun 22; 3:17038. doi: 10.1038/nrdp.2017.38

21. DOMMISH, H. K. Periodontite crônica. In: CARRANZA, Newman. Periodontia clínica, 12. ed. Rio de janeiro: Elsevier, 2016.

22. COSTA, R. P. Nova Classificação das Doenças e Condições Periodontais Um Algoritmo de Diagnóstico. 2019. 48 f. Dissertação (Mestrado). Faculdade de Medicina Dentária Universidade do Porto.

23. NEGRÃO, J. A. da S.; VIANA, J. A. Relação do Mecanismo Patogênico entre Diabetes e Doença Periodontal. RSM - Revista Saúde Multidisciplinar. [periódico online]. 2019.2; 6 $\mathrm{Ed}$.

24. CATON, J. G.; ARMITAGE, G.; BERGLUNDH, T.; CHPPLE, I. L. C. JEPSEN, S.; KORNMAN, K. S.; MEALEY, B. L.; PAPAPANOU, P. N.; SANZ, M.; TONETTI, M. S. A new classification scheme for periodontal and peri-implant diseases and conditions Introduction and key changes from the 1999 classification. Journal of Clinical Periodontology. [periódico online].2018;45(S20):S1-S8.

25. LANG, N. P.; BARTOLD, P. M. Periodontal health. Journal of periodontology. [periódico online]. 2018;89 Suppl 1:S9-s16.

26. GONZALEZ, A. C.; COSTA, T. F.; ANDRADE, Z. A.; MEDRADO, A. R. Wound healing - A literature review. Anais Brasileiros de Dermatologia. [periódico online]. 2016; v. 91, n.5, p. 614-620. set/ out.

27. FIALHO P. G. V; ARAUJO P. H. P. A; ARAUJO M. A. R. Cuidados no Atendimento Odontológico do Paciente Portador de Diabetes Mellitus. Rev. Ciên. Saúde. São Luiz. [periódico online]. 2012. v.14.

28. SILVA, D. F. B.; SILVA, J. E. da; SOUZA E. V. B. de; ALBUQUERQUE, C. R. J. de; CATÃO, M. H. C. de V. Alterações bucais decorrentes do Diabetes Mellitus Tipo 2. FOL -Faculdade de Odontologia de Lins/Unimep. [periódico online]. 2017. 27(2) 27-35. jul.-dez. ISSN Impresso 0104-7582. ISSN Eletrônico: 2238-1236.

29. OLIVEIRA, L. M. L. de; BARBOSA, L. M.; NEGREIROS, J. H. C. N. PENTEADO, L. A. M.; LIMA, V. V. da S. S. Cuidado integrado do paciente periodontal diabético tipo 2 na Atenção Básica: revisão scoping. Physis: Revista de Saúde Coletiva, Rio de Janeiro. [periódico online]. 2020. v. 30(3), e300327. 